\title{
Common Fixed Point for Weakly Compatible Maps in Complete Metric Spaces
}

\author{
M. L. Joshi \\ Associate Professor \\ Department of Mathematics \\ M. \& N. Virani Science College \\ Rajkot. ( INDIA)
}

\author{
Jay G. Mehta \\ Research Scholar (JRF) \\ Harish Chandra Research Institute \\ Allahabad. (IINDIA)
}

\begin{abstract}
In this paper the concept of weakly compatible map in complete metric space has been applied to prove common fixed point theorem for four mappings satisfying implicit relations.
\end{abstract}

\section{Mathematics Subject Classification 47H10, 54H25.}

\section{Keywords}

Common fixed point, complete metric space, compatible maps, weakly compatible maps.

\section{INTRODUCTION}

The study of common fixed point of mappings satisfying contractive type conditions has been a very active field of research activity during the last three decades. In 1922, the Polish mathematician, Banach, proved a theorem which ensures, under appropriate conditions, the existence and uniqueness of a fixed point. His result is called Banach's fixed point theorem or the Banach contraction principle. This theorem provides a technique for solving a variety of applied problems in mathematical science and engineering. Many authors have extended, generalized and improved Banach's fixed point theorem in different ways. In [2], Jungck introduced more generalized commuting mappings, called compatible mappings, which are more general than commuting and weakly commuting mappings. The concept of the commutativity has generalized in several ways. For this Sessa S [6] has introduced the concept of weakly commuting and Gerald Jungck [2] initiated the concept of compatibility. In 1998, Jungck and Rhoades [4] introduced the notion of weakly compatible and showed that compatible maps are weakly compatible but not conversely. Brian Fisher [1] proved an important Common Fixed Point theorem.

The aim of the present paper is to prove a common fixed point theorem on complete metric spaces. Throughout this paper, let $(X, d)$ be a complete metric space unless mentioned otherwise.

\section{PRELIMINARIES}

We recall some definitions and known results.

Definition 2.1. A sequence $\left\{x_{n}\right\}$ in a metric space $(X, d)$ is said to be convergent to a point $x \in X$, denoted by $\lim _{n \rightarrow \infty} x_{n}=x$, if $\lim _{n \rightarrow \infty} d\left(x_{n}, x\right)=0$.
Definition 2.2. A sequence $\left\{x_{n}\right\}$ in a metric space $(X, d)$ is said to be Cauchy sequence if $\lim _{t \rightarrow \infty} d\left(x_{n}, x_{m}\right)=0$ for all $n, m>t$.

Definition 2.3. A metric space $(X, d)$ is said to be complete if every Cauchy sequence in $X$ is convergent.

Remark 2.1. In general a convergent sequence in a metric space $(X, d)$ need not be Cauchy but every convergent sequence is a Cauchy sequence whenever metric $d$ is continuous. A metric $d$ on a set $X$ is said to be weakly continuous if every convergent sequence under $d$ is Cauchy.

Definition 2.4. [2] Let $S$ and T be mappings from a metric space $(X, d)$ into itself. The mappings $S$ and $T$ are said to be compatible if $\lim _{n \rightarrow \infty} d\left(S T x_{n}, T S x_{n}\right)=0$, whenever $\left\{x_{n}\right\}$ is a sequence in $X$ such that $\lim _{n \rightarrow \infty} S x_{n}=\lim _{n \rightarrow \infty} T x_{n}=t$ for some $t \in X$.

Definition 2.5. [4] A pair of self mappings $S$ and $T$ of a metric space $(X, d)$ is said to be weakly compatible if $S x=T x$ (for some $x \in X$ ) implies $S T x=T S x$.

Definition 2.7. A pair $(S, T)$ of self-mappings of a metric space is said to be semi-compatible if $\lim _{n \rightarrow \infty} S T x_{n}=T x$; whenever $\left\{x_{n}\right\}$ is a sequence in $X$ such that $\lim _{n \rightarrow \infty} S x_{n}=\lim _{n \rightarrow \infty} T x_{n}=x$.

Proposition 2.1. Let $(S, T)$ be a compatible pair of self maps on a metric space $(X, d)$ and $T$ be continuous. Then the pair $(S, T)$ is weakly compatible.

It is noted that a compatible maps are weakly compatible but weakly compatible maps need not be compatible.

The converse is not true as seen in following example. 
Example 2.1 Let $x=[0,2]$ with usual metric $d$ where $d(x, y)=|x-y|$ for all $x$ and $y$ in $X$. Let for all $x$ and $y$ in $X$ and $t>0$,

Define:

$T(x)=\left\{\begin{array}{l}x ; x \in[0,1) \\ 2 ; x \in[1,2]\end{array}\right.$ and $S(x)=\left\{\begin{array}{l}2-x ; x \in[0,1) \\ 2 ; x \in[1,2]\end{array}\right.$

Let $x_{n}=1-\frac{1}{n}$ then $T x_{n}=1-\frac{1}{n}$ and $S x_{n}=1+\frac{1}{n}$

Also $T S x_{n}=2$ and $S T x_{n}=1+\frac{1}{n}$.

Thus $\lim _{n \rightarrow \infty} T x_{n}=1$ and $\lim _{n \rightarrow \infty} S x_{n}=1$ and hence $t=1$.

But $\lim _{n \rightarrow \infty} d\left(S T x_{n}, T S x_{n}\right)=\lim _{x \rightarrow \infty}\left|2-\left(1+\frac{1}{n}\right)\right|=1 \neq 0$

Hence $S$ and $T$ are not compatible.

Again $\lim _{n \rightarrow \infty} d\left(S T x_{n}, T S x_{n}\right)=\lim _{x \rightarrow \infty}\left|2-\left(1+\frac{1}{n}\right)\right|=1 \neq 0$

Now we will show that the pair $(S, T)$ is weakly compatible.

Now coincidence points of $S$ and $T$ are in $[1,2]$.

Therefore for any $\mathrm{x}$ in $[1,2]$, we have

$T x=S x=2$ and $T S x=2=S T x$ and $T(2)=2=S(2)$

Thus $(S, T)$ is weakly compatible.

\section{MAIN RESULT.}

\subsection{Implicit Relations}

Let $\mathbf{F}^{*}$ be the set of real functions $\mathrm{F}\left(\mathrm{t}_{1}, \ldots \mathrm{t}_{5}\right):[0, \infty)^{5} \rightarrow[0, \infty)$ satisfying the following conditions :

$\left(F_{1}\right) \quad F$ is non increasing in variables $t_{4}$ and $t_{5}$.

$\left(\mathrm{F}_{2}\right) \quad$ There is an $\mathrm{h}_{1}>0$ and $\mathrm{h}_{2}>0$ such that $\mathrm{h}=\mathrm{h}_{1} \mathrm{~h}_{2}<1$ and if $\mathrm{u} \geq 0, \mathrm{v} \geq 0$ satisfy

$$
\begin{aligned}
\left(\mathrm{F}_{\mathrm{a}}\right) \quad \mathrm{u} & \leq \mathrm{F}(\mathrm{v}, \mathrm{v}, \mathrm{u}, \mathrm{u}+\mathrm{v}, 0) \text { or } \\
\mathrm{u} & \leq \mathrm{F}(\mathrm{v}, \mathrm{u}, \mathrm{v}, \mathrm{u}+\mathrm{v}, 0)
\end{aligned}
$$

then we have $\mathrm{u} \leq \mathrm{h}_{1} \mathrm{v}$.

and if $\mathrm{u} \geq 0, \mathrm{v} \geq 0$ satisfy

$\left(\mathrm{F}_{\mathrm{b}}\right) \quad \mathrm{u} \leq \mathrm{F}(\mathrm{v}, \mathrm{v}, \mathrm{u}, 0, \mathrm{u}+\mathrm{v})$ or $\mathrm{u} \leq \mathrm{F}(\mathrm{v}, \mathrm{u}, \mathrm{v}, 0, \mathrm{u}+\mathrm{v})$

then we have $\mathrm{u} \leq \mathrm{h}_{2} \mathrm{v}$.

$\left(\mathrm{F}_{3}\right)$

$$
\begin{aligned}
& \text { If } \mathrm{u} \geq 0 \text { is such that } \quad \mathrm{u} \leq \mathrm{F}(\mathrm{u}, 0,0, \mathrm{u}, \mathrm{u}) \text { or } \\
& \mathrm{u} \leq \mathrm{F}(0, \mathrm{u}, 0,0, \mathrm{u}) \text { or } \\
& \mathrm{u} \leq \mathrm{F}(0,0, \mathrm{u}, \mathrm{u}, 0)
\end{aligned}
$$

then $\mathrm{u}=0$.

\subsection{Fixed Point Theorem}

Let $\mathrm{S}, \mathrm{T}, \mathrm{I}$ and $\mathrm{J}$ be self-mappings of a complete metric space $(\mathrm{X}, \mathrm{d})$ satisfying the following conditions:
(a) $\mathrm{S}(\mathrm{X}) \subset \mathrm{J}(\mathrm{X}), \mathrm{T}(\mathrm{X}) \subset \mathrm{I}(\mathrm{X})$.
(b) $\mathrm{d}(\mathrm{Sx}, \mathrm{Ty}) \leq$

F(d(Ix, Jy); d(Ix, Sx); d(Jy, Ty); d(Ix, Ty); d(Sx, Jy))

For all $\mathrm{x}$ and $\mathrm{y}$ in $\mathrm{X}$ where $\mathrm{F} \in \mathrm{F}^{*}$.

Then $\mathrm{S}, \mathrm{T}, \mathrm{I}$ and $\mathrm{J}$ have unique common fixed point $\mathrm{Z}$ in $\mathrm{X}$. Further $\mathrm{z}$ is the unique common fixed point of $\mathrm{S}$ and $\mathrm{I}$ and of $\mathrm{T}$ and $\mathrm{J}$.

Proof

$$
\text { let } \mathrm{x}_{0} \in \mathrm{X}
$$

Since $\mathrm{S}(\mathrm{X}) \subset \mathrm{J}(\mathrm{X}), \mathrm{T}(\mathrm{X}) \subset \mathrm{I}(\mathrm{X})$

we can choose $x_{2 n}, x_{2 n+1}$ and $x_{2 n+2}$ such that $\mathrm{Sx}_{2 \mathrm{n}}=\mathrm{Jx}_{2 \mathrm{n}+1}$ and $\mathrm{Tx}_{2 \mathrm{n}+1}=\mathrm{I}_{2 \mathrm{n}+2}, \mathrm{n}=0,1,2 \ldots$

Using (b) we have

$\mathrm{d}\left(\mathrm{Sx}_{2 \mathrm{n}}, \mathrm{Tx}_{2 \mathrm{n}+1}\right)$

$\leq \mathrm{F}\left(\mathrm{d}\left(\mathrm{Ix}_{2 \mathrm{n}}, \mathrm{Jx}_{2 \mathrm{n}+1}\right) ; \mathrm{d}\left(\mathrm{Ix}_{2 \mathrm{n}}, \mathrm{Sx}_{2 \mathrm{n}}\right) ; \mathrm{d}\left(\mathrm{Jx}_{2 \mathrm{n}+1}, \mathrm{Tx}_{2 \mathrm{n}+1}\right) ;\right.$ $\left.\mathrm{d}\left(\mathrm{Ix}_{2 \mathrm{n}}, \mathrm{Tx}_{2 \mathrm{n}+1}\right) ; \mathrm{d}\left(\mathrm{Sx}_{2 \mathrm{n}}, \mathrm{Jx}_{2 \mathrm{n}+1}\right)\right)$

$=\mathrm{F}\left(\mathrm{d}\left(\mathrm{Tx}_{2 \mathrm{n}-1}, \mathrm{Sx}_{2 \mathrm{n}}\right) ; \mathrm{d}\left(\mathrm{Tx}_{2 \mathrm{n}-1}, \mathrm{Sx}_{2 \mathrm{n}}\right) ; \mathrm{d}\left(\mathrm{Sx}_{2 \mathrm{n}}, \mathrm{Tx}_{2 \mathrm{n}+1}\right)\right.$; $\left.\mathrm{d}\left(\mathrm{Tx}_{2 \mathrm{n}-1}, \mathrm{Tx}_{2 \mathrm{n}+1}\right) ; 0\right)$

$\leq \mathrm{F}\left(\mathrm{d}\left(\mathrm{Sx}_{2 \mathrm{n}}, \mathrm{Tx}_{2 \mathrm{n}-1}\right) ; \mathrm{d}\left(\mathrm{Sx}_{2 \mathrm{n}}, \mathrm{Tx}_{2 \mathrm{n}-1}\right) ; \mathrm{d}\left(\mathrm{Sx}_{2 \mathrm{n}}, \mathrm{Tx}_{2 \mathrm{n}+1}\right)\right.$; $\left.\mathrm{d}\left(\mathrm{Tx}_{2 \mathrm{n}-1}, \mathrm{Tx}_{2 \mathrm{n}+1}\right) ; 0\right)$

$\leq \mathrm{F}\left(\mathrm{d}\left(\mathrm{Sx}_{2 \mathrm{n}}, \mathrm{Tx}_{2 \mathrm{n}-1}\right) ; \mathrm{d}\left(\mathrm{Sx}_{2 \mathrm{n}}, \mathrm{Tx}_{2 \mathrm{n}-1}\right) ; \mathrm{d}\left(\mathrm{Sx}_{2 \mathrm{n}}, \mathrm{Tx}_{2 \mathrm{n}+1}\right)\right.$; $\left.\mathrm{d}\left(\mathrm{Sx}_{2 \mathrm{n}}, \mathrm{Tx}_{2 \mathrm{n}-1}\right)+\mathrm{d}\left(\mathrm{Sx}_{2 \mathrm{n}}, \mathrm{Tx}_{2 \mathrm{n}+1}\right) ; 0\right)$

Thus by property $\left(\mathrm{F}_{\mathrm{a}}\right), \mathrm{d}\left(\mathrm{Sx}_{2 \mathrm{n}}, \mathrm{Tx}_{2 \mathrm{n}+1}\right) \leq \mathrm{h}_{1} \mathrm{~d}\left(\mathrm{Sx}_{2 \mathrm{n}}, \mathrm{Tx}_{2 \mathrm{n}-1}\right)$. Similarly, $\quad \mathrm{d}\left(\mathrm{Tx}_{2 \mathrm{n}-1}, \mathrm{Sx}_{2 \mathrm{n}}\right) \leq \mathrm{h}_{2} \mathrm{~d}\left(\mathrm{Sx}_{2 \mathrm{n}-2}, \mathrm{Tx}_{2 \mathrm{n}-1}\right)$. Therefore $\quad \mathrm{d}\left(\mathrm{Sx}_{2 \mathrm{n}}, \mathrm{Tx}_{2 \mathrm{n}+1}\right) \leq \mathrm{hd}\left(\mathrm{Sx}_{2 \mathrm{n}-2}, \mathrm{Tx}_{2 \mathrm{n}-1}\right)$. From this we can deduce that $\mathrm{d}\left(\mathrm{Sx}_{2 \mathrm{n}}, \mathrm{Tx}_{2 \mathrm{n}+1}\right) \leq \mathrm{h}^{\mathrm{n}} \mathrm{d}\left(\mathrm{Sx}_{0}, \mathrm{~T}_{1}\right)$. $\mathrm{d}\left(\mathrm{Tx}_{2 \mathrm{n}+1}, \mathrm{Sx}_{2 \mathrm{n}+2}\right) \leq \mathrm{h}_{2} \mathrm{~h}^{\mathrm{n}} \mathrm{d}\left(\mathrm{Sx}_{0}, \mathrm{~T}_{1}\right)$

for $\mathrm{n}=1,2, \ldots \ldots$

Since $\mathrm{h}<1$, the sequence

$\left\{\mathrm{Sx}_{0}, \mathrm{Tx}_{1}, \mathrm{Sx}_{2}, \ldots . \mathrm{Tx}_{2 \mathrm{n}-1}, \mathrm{Sx}_{2 \mathrm{n}}, \mathrm{Tx}_{2 \mathrm{n}+1}, \ldots\right\}$

is a Cauchy sequence.

Since $(X, d)$ is complete metric space, this sequence has a limit $\mathrm{Z}$ in $\mathrm{X}$ and the subsequences

$\left\{\mathrm{Sx}_{2 \mathrm{n}}\right\}=\left\{\mathrm{Jx}_{2 \mathrm{n}+1}\right\}$ and $\left\{\mathrm{Tx}_{2 \mathrm{n}+1}\right\}=\left\{\mathrm{I}_{2 \mathrm{n}+2}\right\}$ converge to the point $\mathrm{z}$.

We suppose that the mapping $\mathrm{I}$ is continuous, so that the sequences $\left\{\mathrm{I}^{2} \mathrm{x}_{2 n}\right\}$ and $\left\{\mathrm{ISx}_{2 \mathrm{n}}\right\}$ converge to the point Iz. Since $\mathrm{S}$ and I are weakly commute, we have $\mathrm{d}\left(\mathrm{ISx}_{2 \mathrm{n}}, \mathrm{SIx}_{2 \mathrm{n}}\right) \leq \mathrm{d}\left(\mathrm{Ix}_{2 \mathrm{n}}, \mathrm{Sx}_{2 \mathrm{n}}\right)$

So that the sequence $\left\{\mathrm{SIx}_{2 \mathrm{n}}\right\}$ converges to the point Iz. Using (b) we have,

$$
\begin{aligned}
& \mathrm{d}\left(\mathrm{SIx}_{2 \mathrm{n}}, \mathrm{Tx}_{2 \mathrm{n}+1}\right) \\
& \leq \mathrm{F}\left(\mathrm{d}\left(\mathrm{I}^{2} \mathrm{x}_{2 \mathrm{n}}, \mathrm{JX}_{2 \mathrm{n}+1}\right) ; \mathrm{d}\left(\mathrm{I}^{2} \mathrm{x}_{2 \mathrm{n}}, \mathrm{SIx}_{2 \mathrm{n}}\right) ; \mathrm{d}\left(\mathrm{Jx}_{2 \mathrm{n}+1}, \mathrm{Tx}_{2 \mathrm{n}+1}\right) ;\right. \\
& \left.\mathrm{d}\left(\mathrm{I}^{2} \mathrm{X}_{2 \mathrm{n}}, \mathrm{Tx}_{2 \mathrm{n}+1}\right) ; \mathrm{d}\left(\mathrm{SIx}_{2 \mathrm{n}}, \mathrm{JX}_{2 \mathrm{n}+1}\right)\right)
\end{aligned}
$$

By letting $n \rightarrow \infty$, we get

$$
\mathrm{d}(\mathrm{Iz}, \mathrm{z}) \leq \mathrm{F}(\mathrm{d}(\mathrm{Iz}, \mathrm{z}) ; 0 ; 0 ; \mathrm{d}(\mathrm{Iz}, \mathrm{z}) ; \mathrm{d}(\mathrm{Iz}, \mathrm{z}))
$$

Therefore by property $\left(\mathrm{F}_{3}\right)$, we get $\mathrm{d}(\mathrm{Iz}, \mathrm{z})=0$, i.e. $\mathrm{Iz}=\mathrm{z}$. Again by using (b) we have,

$$
\begin{gathered}
\mathrm{d}\left(\mathrm{Sz}_{2} \mathrm{Tx}_{2 \mathrm{n}+1}\right) \leq \mathrm{F}\left(\mathrm{d}\left(\mathrm{Iz}, \mathrm{Jx}_{2 \mathrm{n}+1}\right) ; \mathrm{d}(\mathrm{Iz}, \mathrm{Sz}) ; \mathrm{d}\left(\mathrm{Jx}_{2 \mathrm{n}+1}, \mathrm{Tx}_{2 \mathrm{n}+1}\right) ;\right. \\
\left.\mathrm{d}\left(\mathrm{Iz}, \mathrm{Tx}_{2 \mathrm{n}+1}\right) ; \mathrm{d}\left(\mathrm{Sz}_{\mathbf{J}} \mathrm{Jx}_{2 \mathrm{n}+1}\right)\right)
\end{gathered}
$$

By letting $n \rightarrow \infty$, we get

$$
\mathrm{d}(\mathrm{Sz}, \mathrm{z}) \leq \mathrm{F}(0 ; \mathrm{d}(\mathrm{z}, \mathrm{Sz}) ; 0 ; 0 ; \mathrm{d}(\mathrm{Sz}, \mathrm{z}))
$$

Therefore by property $\left(\mathrm{F}_{3}\right)$, we get $\mathrm{d}(\mathrm{Sz}, \mathrm{z})=0$, i.e. $\mathrm{Sz}=\mathrm{z}$. Since $S(X) \subset J(X)$, there is a point $y$ in $X$ such that $\mathrm{Jy}=\mathrm{z}$. Therefore by (b), we have $\mathrm{d}(\mathrm{z}, \mathrm{Ty})=\mathrm{d}(\mathrm{Sz}, \mathrm{Ty}) \leq \mathrm{F}(\mathrm{d}(\mathrm{Iz}, \mathrm{Jy}) ; \mathrm{d}(\mathrm{Iz}, \mathrm{Sz}) ; \mathrm{d}(\mathrm{Jy}, \mathrm{Ty}) ;$ $\mathrm{d}(\mathrm{Iz}, \mathrm{Ty}) ; \mathrm{d}(\mathrm{Sz}, \mathrm{Jy}))$

so that $\mathrm{d}(\mathrm{z}, \mathrm{Ty}) \leq \mathrm{F}(0 ; 0 ; \mathrm{d}(\mathrm{z}, \mathrm{Ty}) ; \mathrm{d}(\mathrm{z}, \mathrm{Ty}) ; 0)$ Therefore by property $\left(F_{3}\right)$, we get $d(z, T y)=0$, i.e. $T y=z$. Since $\mathrm{T}$ and $\mathrm{J}$ are weakly commute, we have 
$\mathrm{d}(\mathrm{Tz}, \mathrm{Jz})=\mathrm{d}(\mathrm{TJy}, \mathrm{JTy}) \leq \mathrm{d}(\mathrm{Jy}, \mathrm{Ty})=0$

Thus $\mathrm{Tz}=\mathrm{Jz}$ and so that by (b), we have

$$
\begin{aligned}
\mathrm{d}(\mathrm{z}, \mathrm{Tz})=\mathrm{d}(\mathrm{Sz}, \mathrm{Tz}) & \leq \mathrm{F}(\mathrm{d}(\mathrm{Iz}, \mathrm{Jz}) ; \mathrm{d}(\mathrm{Iz}, \mathrm{Sz}) ; \mathrm{d}(\mathrm{Jz}, \mathrm{Tz}) ; \mathrm{d}(\mathrm{Iz}, \mathrm{Tz}) ; \\
& =\mathrm{F}(\mathrm{dz}(\mathrm{z}, \mathrm{Tz})) \\
& \quad \mathrm{d}(\mathrm{z}, \mathrm{d}(\mathrm{z}, \mathrm{zz}) ; \mathrm{d}(\mathrm{Tz}, \mathrm{Tz}) ; \mathrm{d}(\mathrm{z}, \mathrm{Tz}) ; \\
= & \mathrm{F}(\mathrm{d}(\mathrm{z}, \mathrm{Tz}) ; 0 ; 0 ; \mathrm{d}(\mathrm{z}, \mathrm{Tz}) ; \mathrm{d}(\mathrm{z}, \mathrm{Tz}))
\end{aligned}
$$

Therefore by property $\left(\mathrm{F}_{3}\right)$, we get $\mathrm{d}(\mathrm{z}, \mathrm{Tz})=0$,

i.e. $\mathrm{Tz}=\mathrm{z}$ i.e. $\mathrm{z}=\mathrm{Tz}=\mathrm{Jz}$.

Since $\mathrm{Iz}=\mathrm{Sz}=\mathrm{z}$, we get $\mathrm{z}=\mathrm{Tz}=\mathrm{Jz}=\mathrm{Iz}=\mathrm{Sz}$

Thus $z$ is a common fixed point of $S, T, I$ and $J$.

On the other way the proof is similar if mapping $\mathrm{J}$ is continuous.

Now if we consider that the mapping $\mathrm{S}$ or $\mathrm{T}$ is continuous, in the similar way we can prove that $\mathrm{z}$ is a common fixed point of $\mathrm{S}, \mathrm{T}$, I and J.

Uniqueness : For uniqueness let us suppose that there is another fixed point $\mathrm{u}$ of $\mathrm{S}$ and $\mathrm{I}$.

Therefore by (b), we have

$\mathrm{d}(\mathrm{Su}, \mathrm{Tz})=\mathrm{d}(\mathrm{u}, \mathrm{z}) \leq \mathrm{F}(\mathrm{d}(\mathrm{Iu}, \mathrm{Jz}) ; \mathrm{d}(\mathrm{Iu}, \mathrm{Su}) ; \mathrm{d}(\mathrm{Jz}, \mathrm{Tz}) ; \mathrm{d}(\mathrm{Iu}, \mathrm{Tz}) ;$ $\mathrm{d}(\mathrm{Su}, \mathrm{Jz}))$

$=\mathrm{F}(\mathrm{d}(\mathrm{u}, \mathrm{z}) ; 0 ; 0 ; \mathrm{d}(\mathrm{u}, \mathrm{z}) ; \mathrm{d}(\mathrm{u}, \mathrm{z}))$

Therefore by property $\left(\mathrm{F}_{3}\right)$, we get $\mathrm{d}(\mathrm{u}, \mathrm{z})=0$, i.e. $\mathrm{u}=\mathrm{z}$.

Similarly it can be proved that $\mathrm{z}$ is the unique common fixed point of $\mathrm{T}$ and $\mathrm{J}$.

Hence the theorem.

\section{Remark}

Let $\mathbf{G}^{*}$ be the set of real functions $\mathrm{G}\left(\mathrm{t}_{1}, \mathrm{t}_{2}, \mathrm{t}_{3}\right):[0, \infty)^{3} \rightarrow[0, \infty)$ satisfying the following conditions :

$\left(\mathrm{G}_{1}\right) \quad \mathrm{G}(1,1,1)=\mathrm{h}<1$.

$\left(\mathrm{G}_{2}\right) \quad$ If $\mathrm{u} \geq 0, \mathrm{v} \geq 0$ be such that $\mathrm{u} \leq \mathrm{G}(\mathrm{u}, \mathrm{u}, \mathrm{u})$ or

$$
\begin{aligned}
\mathrm{u} & \leq \mathrm{G}(\mathrm{v}, \mathrm{v}, \mathrm{u}) \text { or } \\
\mathrm{u} & \leq \mathrm{G}(\mathrm{v}, \mathrm{u}, \mathrm{v})
\end{aligned}
$$

then we have $\mathrm{u} \leq \mathrm{hv}$.

It should be noted that $\mathrm{G}^{*} \subset \mathrm{F}^{*}$ but $\mathrm{G}^{*} \neq \mathrm{F}^{*}$.

Corollary Let S, T, I and $\mathrm{J}$ be self-mappings of a complete metric space $(X, d)$ satisfying the following conditions:

(a) $\mathrm{S}(\mathrm{X}) \subset \mathrm{J}(\mathrm{X}), \mathrm{T}(\mathrm{X}) \subset \mathrm{I}(\mathrm{X})$.

(b) $\mathrm{d}(\mathrm{Sx}, \mathrm{Ty}) \leq \mathrm{G}(\mathrm{d}(\mathrm{Ix}, \mathrm{Jy}) ; \mathrm{d}(\mathrm{Ix}, \mathrm{Sx}) ; \mathrm{d}(\mathrm{Jy}, \mathrm{Ty}))$

For all $\mathrm{x}$ and $\mathrm{y}$ in $\mathrm{X}$ where $\mathrm{G} \in \mathbf{G}^{*}$.
Then $\mathrm{S}, \mathrm{T}$, I and $\mathrm{J}$ have unique common fixed point $\mathrm{Z}$ in $\mathrm{X}$. Further $\mathrm{z}$ is the unique common fixed point of $\mathrm{S}$ and $\mathrm{I}$ and of $\mathrm{T}$ and $\mathrm{J}$.

Proof : Proof is follows form the theorem because $G^{*} \subset F^{*}$.

\section{ACKNOWLEDGMENTS}

We are thankful to Prof. L. N. Joshi and Prof. J. N. Chauhan for their cooperation in the preparation of this paper. We are also thankful to the numerous referees for their helpful and valuable comments.

\section{REFERENCES}

[1] B.Fisher, "Common Fixed Point of Four Mappings", Bull. Inst. of .Math. Academia. Sinicia, vol.11, 1983, pp.103-113.

[2] Jungck, G., Compatible mappings and common fixed points, Internet. I. Math and Math. Sci. 9 (1986), 771-779.

[3] G. Jungck, P. P. Murthy and Y. J. Cho, Compatible mappings of type (A) and common fixed points, Math. Japonica, 38, (1993), 381-386.

[4] G.Jungck, and B.E.Rhoades," Fixed Point for set valued Functions Without Continuity”, Indian J. Pure Appl. Math, vol.29,no.3, 1998, pp.227-238.

[5] V. Sriniwas and R. Uma Maheswar Rao, A Common Fixed Point Theorem for Four Selfmaps, The Mathematics Education, Vol. XL, No. 2, June. (2006), pp 99-104.

[6] S.Sessa, "On a weak Commutativity Condition of Mappings in a Fixed Point Considerations", Publ. Inst Math. Debre., Vol.32,1982, pp.149-153.

[7] Brown, L. D., Hua, H., and Gao, C. 2003. A widget framework for augmented interaction in SCAPE.

[8] V. Popa, A general fixed point theorem for four weakly compatible mappings satisfying an implicit relation, Filomat 19 (2005), 45-51.

[9] R. Chugh and S. Kumar, Common fixed points for weakly compatible maps, Proc. Indian Acad. Sci. Math. Sci. 111 (2) (2001), 241-247. 